\title{
Bounds for the Spectral Radius of a Matrix
}

\author{
By N. A. Derzko and A. M. Pfeffer
}

Let $A=\left[a_{i j}\right]$ be an $n \times n$ matrix with complex entries. We define $\rho(A)$ to be the spectral radius of $A$ and $|A|$ to be the matrix $\left[\left|a_{i j}\right|\right]$.

A. Brauer [1], W. Ledermann [2] and A. Ostrowski [4] have developed bounds for $\rho(|A|)$. Their results, coupled with the result of Perron and Frobenius [6] that $\rho(A) \leqq \rho(|A|)$ give upper bounds for $\rho(A)$ which are not less than $\rho(|A|)$. These bounds are restricted to matrices with nonzero entries and do not take into account the effect of the phases of the entries of $A$ on $\rho(A)$. In Section I of this paper we obtain a sequence of bounds for $\rho(A)$ in terms of $\rho\left(\left|A^{r}\right|\right)(r=1,2, \cdots)$ which are less than or equal to $\rho(|A|)$ and converge to $\rho(A)$. In this manner we are partially accounting for the effect on $\rho(A)$ of the phases of the $a_{i j}$. In Section II we derive bounds for $\rho(A)$ in terms of the Frobenius norm of $A$. These bounds always lie in the field of values of $A$, are computationally well suited to complex matrices and 'can be used in conjunction with the techniques of Section I.

The authors are indebted to Olga Taussky and Alston Householder for suggestions.

I. Bounds for $\rho(A)$. Let $a_{j k}=\left|a_{j k}\right| \exp \left(i \theta_{j k}\right)$, where $0 \leqq \theta_{j k}<2 \pi$. We define

$$
\omega_{k}=\left[\rho\left(\left|A^{k}\right|\right)\right]^{1 / k}, \quad k=1,2, \cdots .
$$

Lemma 1. If $k$ and $r$ are positive integers, then $\omega_{k r} \leqq \omega_{k}$.

Proof. Since $0 \leqq\left|A^{k r}\right| \leqq\left|A^{k}\right|^{r}$, it follows that $\rho\left(\left|A^{k r}\right|\right) \leqq \rho\left(\left|A^{k}\right|^{r}\right)$. We have always $\rho\left(\left|A^{k}\right|^{r}\right)=\left[\rho\left(\left|A^{k}\right|\right)\right]^{r}$. Consequently,

$$
\left[\rho\left(\left|A^{k r}\right|\right)\right]^{1 / k r} \leqq\left[\rho\left(\left|A^{k}\right|\right)\right]^{1 / k}
$$

or $\omega_{k r} \leqq \omega_{k}$.

In particular, we deduce

$$
\omega_{r} \leqq \omega_{1}=\rho(|A|), \quad r=1,2, \cdots .
$$

LEMMA 2. The $\omega_{k}(k=1,2, \cdots)$ form a sequence of upper bounds for $\rho(A)$ which converges to $\rho(A)$.

Proof. Since $\rho\left(A^{k}\right) \leqq \rho\left(\left|A^{k}\right|\right)$, it follows that $\rho(A) \leqq\left[\rho\left(\left|A^{k}\right|\right)\right]^{1 / k}=\omega_{k}$, which proves our first assertion. To prove convergence of the $\omega_{k}$ we define the multiplicative matrix norm

$$
N(A)=\max _{1 \leqq i \leqq n}\left(\sum_{j=1}^{n}\left|a_{i j}\right|\right),
$$

and use the general results [3] that

$$
\lim _{k \rightarrow \infty}\left[N\left(A^{k}\right)\right]^{1 / k}=\rho(A)
$$

and $[\rho(A)]^{k} \leqq \omega_{k}^{k} \leqq N\left(A^{k}\right)$. Taking $k$ th roots we conclude

$$
\lim _{k \rightarrow \infty} \omega_{k}=\rho(A) .
$$

Received June 29, 1964. This work was supported in part by the Office of Naval Research and by the National Science Foundation. 
Note. In general the $\omega_{k}$ do not decrease monotonically to $\rho(A)$. However, Lemma 1 can be used to obtain decreasing subsequences such as $\omega_{1}, \omega_{2}, \omega_{4}, \omega_{8}, \cdots$.

If $A$ is irreducible, it is known [6] that $\omega_{1}=\rho(A)$ if and only if $A=e^{i \phi} D|A| D^{-1}$, where $D$ is a diagonal matrix whose diagonal entries have modulus unity. If $A$ is of this special form, then $\omega_{1}=\omega_{k}(k=1,2, \cdots)$. Furthermore, if we know all the $\omega_{k}$ are equal, Lemma 2 tells us that $\rho(A)$ has their common value. It is natural to ask what happens in case $\omega_{j}=\omega_{k}$ for some $j$ and $k$.

THEOREM 1. If $A$ has only nonzero entries and if $m>1$, then $\omega_{1}=\omega_{m}$ if and only if $\rho(A)=\omega_{1}$.

Proof. We have already remarked that $\rho(A)=\omega_{1}$ implies $\omega_{1}=\omega_{k}(k=1,2, \cdots)$ and, in particular, $\omega_{1}=\omega_{m}$.

Conversely, suppose $\omega_{1}=\omega_{m}$ for some $m>1$. This means

$$
\rho\left(\left|A^{m}\right|\right)=[\rho(|A|)]^{m}=\rho\left[|A|^{m}\right] .
$$

Since $|A|^{m}$ is a positive matrix and $\left|A^{m}\right| \leqq|A|^{m}$, the Perron-Frobenius theory tells us that $|A|^{m}=\left|A^{m}\right|$. If we write out the expressions for the $j, k$ th entries of $|A|^{m}$ and $\left|A^{m}\right|$, and use the fact that the modulus of a sum of complex numbers equals the sum of their moduli only when the numbers have the same arguments, we obtain the equation

$$
\theta_{j l_{1}}+\theta_{l_{1} l_{2}}+\cdots+\theta_{l_{m-1} k} \equiv \alpha_{j k} .
$$

Here, and elsewhere, congruences are modulo $2 \pi ; \alpha_{j k}$ is the argument of the $j, k$ th entry of $A^{m}$ and is independent of the indices $l_{1}, \cdots, l_{m-1}, 1 \leqq l_{i} \leqq n(i=1, \cdots$, $m-1)$. In particular,

$$
\alpha_{11} \equiv \theta_{1 j}+\theta_{j 1}+\theta_{11}+\cdots+\theta_{11}=\theta_{j 1}+\theta_{11}+\cdots+\theta_{11}+\theta_{1 j} \equiv \alpha_{j j} .
$$

Similarly,

and

$$
\alpha_{i j} \equiv \theta_{i 1}+\theta_{11}+\cdots+\theta_{11}+\theta_{1 j},
$$

$$
\alpha_{j k} \equiv \theta_{j 1}+\theta_{11}+\cdots+\theta_{11}+\theta_{1 k} .
$$

Therefore,

$$
\begin{aligned}
\alpha_{i j}+\alpha_{j k} & \equiv \theta_{i 1}+\theta_{11}+\cdots+\theta_{11}+\theta_{1 k}+\theta_{j 1}+\theta_{11}+\cdots+\theta_{11}+\theta_{1 j} \\
& \equiv \alpha_{i k}+\alpha_{j j} \equiv \alpha_{i k}+\alpha_{11} .
\end{aligned}
$$

Let $\delta_{r} \equiv \alpha_{11}-\alpha_{1 r}, 1 \leqq r \leqq n$. Then

$$
\begin{aligned}
\alpha_{i k} & \equiv \alpha_{i j}+\alpha_{j k}-\alpha_{11} \\
& \equiv \alpha_{i 1}+\alpha_{1 k}-\alpha_{11} \\
& \equiv\left(2 \alpha_{11}-\alpha_{1 i}\right)+\alpha_{1 k}-\alpha_{11} \\
& \equiv \delta_{i}-\delta_{k}+\alpha_{11} .
\end{aligned}
$$

Define $D$ to be the matrix

$\operatorname{diag}\left(\exp i \delta_{1}, \cdots, \exp i \delta_{n}\right)$.

Then $A^{m}=\left(\exp i \alpha_{11}\right) D\left|A^{m}\right| D^{-1}$ so that

$$
\rho\left(A^{m}\right)=\rho\left(\left|A^{m}\right|\right)
$$


and

$$
\rho(A)=\omega_{m}=\omega_{1} .
$$

Theorem 2. If $m$ and $r$ are positive integers with $r>1$, and $\left|A^{m}\right|>0$, then $\omega_{m}=\omega_{r m}$ if and only if $\rho(A)=\omega_{m}$.

Proof. Suppose $\omega_{m}=\omega_{r m}$. Then

$$
\left[\rho\left(\left|A^{m}\right|\right)\right]^{1 / m}=\left[\rho\left(\left|A^{r m}\right|\right)\right]^{1 / r m}
$$

and

$$
\left[\rho\left(\left|A^{m}\right|\right)\right]^{r}=\rho\left(\left|A^{r m}\right|\right) .
$$

Since $\left|A^{m}\right|>0$, if we apply Theorem 1 to $A^{m}$, we may conclude that

$$
\rho\left(A^{m}\right)=\rho\left(\left|A^{m}\right|\right) \text {. }
$$

Hence, $\rho(A)=\omega_{m}$.

Conversely, suppose $\rho(A)=\omega_{m}$. By Lemma $1, \omega_{m} \geqq \omega_{r m}$ and, by Lemma 2, $\omega_{r m} \geqq \rho(A)$. Consequently, $\omega_{m}=\omega_{r m}$.

Theorem 1 remains true if we replace the assumption " $A$ has only nonzero entries" by the slightly weaker condition "for some $r$ neither the $r$ th row nor the $r$ th column of $A$ has zero entries and $|A|^{m}>0$." Theorem 2 can be modified analogously. However, the following example shows that in general it is not possible to relax the assumption of Theorem 1 that $A$ is a matrix with only nonzero entries to " $A$ is irreducible." This relaxation is possible in the Perron-Frobenius theory [6] and one is tempted to try it here. Let

$$
A=\left[\begin{array}{rrr}
0 & 1 & 0 \\
-1 & 0 & 1 \\
0 & 1 & 0
\end{array}\right]
$$

Then $A$ is irreducible but $\rho(A)=0$ and $\omega_{1}=\omega_{2}=\sqrt{ } 2$.

In Theorem 2 we proved that the condition $\omega_{i}=\omega_{k}$, where $i<k, i \mid k$, and $\left|A^{i}\right|>0$, is sufficient to ensure $\rho(A)=\omega_{i}$. One would like to eliminate the requirement $i \mid k$; however, examples have been constructed showing that, in general, this is not possible.

The following example shows that in some cases a rough estimate for $\omega_{2}$ is a better bound for $\rho(A)$ than $\omega_{1}$ itself. Let

$$
A=\left[\begin{array}{ll}
2 & -1 \\
1 & -1
\end{array}\right] \text {. }
$$

Then $\rho(A) \approx 1.62, \omega_{1} \approx 2.62$ and $\omega_{2} \approx 1.82$. The square root of the Gerschgorin circle estimated for $\rho\left(\left|A^{2}\right|\right)$ is 2 .

II. Upper Bounds for $\rho(A)$ in terms of $\epsilon(A)$. The Frobenius multiplicative matrix norm $\epsilon(A)[5]$ is defined by

$$
\epsilon(A)=\left[\sum_{i, j=1}^{n}\left|a_{i j}\right|^{2}\right]^{1 / 2} .
$$

Since $\epsilon$ is a multiplicative norm we have $\rho(A) \leqq \epsilon(A)$. The following result gives the condition for equality. 
LEMma 3. The Frobenius norm of $A=\left[a_{j k}\right]$ is its spectral radius if and only if $a_{j k}=e^{i \theta} x_{j} \bar{x}_{k}$, where $\bar{x}_{k}$ denotes the complex conjugate of $x_{k}$ and $0 \leqq \theta<2 \pi$.

Proof. If $a_{j k}=e^{i \theta} x_{j} \bar{x}_{k}(j, k=1, \cdots, n)$, then the only nonzero eigenvalue of $A$ is $e^{i \theta}\left(\sum_{j=1}^{n}\left|x_{j}\right|^{2}\right)$ corresponding to the eigenvector with components $x_{j}$ $(j=1, \cdots, n)$. Furthermore,

$$
\begin{aligned}
{[\epsilon(A)]^{2} } & =\sum_{j, k=1}^{n}\left|x_{j}\right|^{2}\left|x_{k}\right|^{2} \\
& =\left(\sum_{j=1}^{n}\left|x_{j}\right|^{2}\right)^{2}=[\rho(A)]^{2} .
\end{aligned}
$$

On the other hand, suppose $\rho(A)=\epsilon(A)$. We may assume $\rho(A)>0$ since $\epsilon(A)=\rho(A)=0$ implies $A=0$. Let $e^{i \theta} \rho(A)$ be an eigenvalue of maximum modulus, whose associated eigenvector has components $x_{j}(j=1, \cdots, n)$ normalized so that $\rho(A)=\sum_{j=1}^{n}\left|x_{j}\right|^{2}$. We have, by the Cauchy-Schwarz inequality,

$$
\begin{aligned}
\left|e^{i \theta} \rho(A) x_{j}\right|^{2} & =\left|\sum_{k=1}^{n} a_{j k} x_{k}\right|^{2} \\
& \leqq\left(\sum_{k=1}^{n}\left|a_{j k}\right|^{2}\right)\left(\sum_{k=1}^{n}\left|x_{k}\right|^{2}\right), \quad j=1, \cdots, n .
\end{aligned}
$$

In order that $\rho(A)=\epsilon(A)$, equality must hold for each $j$ above, which implies

$$
a_{j k}=\eta_{j} \bar{x}_{k} \quad(j, k=1, \cdots, n),
$$

where the $\eta_{j}$ are constants. Then

$$
e^{i \theta} \rho(A) x_{j}=\sum_{k=1}^{n} \eta_{j} \bar{x}_{k} x_{k}=\eta_{j} \rho(A),
$$

so that $\eta_{j}=e^{i \theta} x_{j}$ and $a_{j k}=e^{i \theta} x_{j} \bar{x}_{k}$, as required.

The following alternate proof of Lemma 3 is due to Alston Householder.

The Frobenius norm is the square root of the sum of the squares of the singular values of $A$, and the largest singular value alone is greater than or equal to the spectral radius. Hence, for equality, the others must be zero implying $A^{*} A$ is of rank 1. Therefore $A$ is also of rank 1 and hence of the form $a b^{*}$ where $a$ and $b$ are column vectors. But the only non-null root of $a b^{*}$ is $b^{*} a$. From $\left[\epsilon\left(a b^{*}\right)\right]^{2}=a^{*} a b^{*} b=$ $\left|b^{*} a\right|^{2}$, we conclude $a$ and $b$ are linearly dependent, from which the result follows.

Ideally, one would wish to develop bounds for $\rho(A)$ which depend on $\epsilon(A)$ and some measure of the departure of $A$ from the special form of Lemma 3 . One approach is to minimize the Frobenius norm of matrices which are similar to $A$.

Define

and

$$
R_{i}=\left[\left(\sum_{j=1}^{n}\left|a_{i j}\right|^{2}\right)-\left|a_{i i}\right|^{2}\right]^{1 / 2}
$$

$$
C_{i}=\left[\left(\sum_{j=1}^{n}\left|a_{j i}\right|^{2}\right)-\left|a_{i i}\right|^{2}\right]^{1 / 2} .
$$

Theorem 3. If $A$ is an $n \times n$ complex matrix, then

$$
[\rho(A)]^{2} \leqq[\epsilon(A)]^{2}-\left[\max _{1 \leqq i \leqq n}\left|R_{i}-C_{i}\right|\right]^{2} .
$$


Proof. We prove the equivalent statement

$$
[\rho(A)]^{2} \leqq[\epsilon(A)]^{2}-\left(R_{i}-C_{i}\right)^{2}, \quad i=1, \cdots, n .
$$

Suppose first that neither $R_{i}$ nor $C_{i}$ is zero. Let $D_{v}$ be the diagonal matrix whose diagonal entries are all unity except for $v \neq 0$ in the $i$ th position. Then $\rho\left(D_{v} A D_{v}{ }^{-1}\right)$ $=\rho(A)$. Hence, $[\rho(A)]^{2} \leqq\left[\epsilon\left(D_{v} A D_{v}{ }^{-1}\right]^{2}=[\epsilon(A)]^{2}-R_{i}{ }^{2}-C_{i}{ }^{2}+v^{2} R_{i}{ }^{2}+v^{-2} C_{i}{ }^{2}\right.$. If we minimize the right-hand expression over $v$ we obtain $v^{2}=C_{i} / R_{i}$, and

$$
[\rho(A)]^{2} \leqq[\epsilon(A)]^{2}-\left(R_{i}-C_{i}\right)^{2} .
$$

Since $\rho(A), \epsilon(A), R_{i}$ and $C_{i}$ all depend continuously on the entries of $A$, it follows that the restriction $R_{i}, C_{i} \neq 0$ can be removed.

If it happens that $R_{i}, C_{i} \neq 0$, where $i$ is the index which gives the maximum in Theorem 3 , then Theorem 3 may be applied to the matrix $D_{v} A D_{v}{ }^{-1}$, where $v^{2}=C_{i} / R_{i}$, giving a possible improvement in the bound for $\rho(A)$.

If $\lambda_{1}, \cdots, \lambda_{n}$ are the eigenvalues of $A$, then it is easily seen that

$$
\inf \left\{\left[\epsilon\left(S A S^{-1}\right)\right]^{2}: S \text { nonsingular }\right\}=\sum_{i=1}^{n}\left|\lambda_{i}\right|^{2} .
$$

Hence, the bound given by Theorem 3 must be greater than or equal to $\sum_{i=1}^{n}\left|\lambda_{i}\right|^{2}$.

We will now consider bounds which in some cases are actually less than $\sum_{i=1}^{n}\left|\lambda_{i}\right|^{2}$. Let $\operatorname{tr} A$ be the trace of $A$.

THEOREM 4. If $A$ is an $n \times n$ complex matrix, then

$$
\rho(A) \leqq(1-1 / n)^{1 / 2}\left\{\left[\epsilon\left(S A S^{-1}\right)\right]^{2}-|\operatorname{tr} A|^{2} / n\right\}^{1 / 2}+|\operatorname{tr} A| / n,
$$

for any nonsingular $S$.

Proof. Let $\lambda_{M}$ be an eigenvalue of maximum modulus. Then, from

$$
\sum_{i=1}^{n}\left|\lambda_{i}\right|^{2} \leqq\left[\epsilon\left(S A S^{-1}\right)\right]^{2}
$$

by an application of the Cauchy-Schwarz inequality we find

$$
\begin{aligned}
\left|\lambda_{M}\right|^{2} & \leqq\left[\epsilon\left(S A S^{-1}\right)\right]^{2}-\sum_{i \neq M}\left|\lambda_{i}\right|^{2} \\
& \leqq\left[\epsilon\left(S A S^{-1}\right)\right]^{2}-\left|\sum_{i \neq M} \lambda_{i}\right|^{2} /(n-1) \\
& =\left[\epsilon\left(S A S^{-1}\right)\right]^{2}-\left|\operatorname{tr} A-\lambda_{M}\right|^{2} /(n-1),
\end{aligned}
$$

from which it follows, by elementary means, that

$$
\left|\lambda_{M}\right| \leqq(1-1 / n)^{1 / 2}\left\{\left[\epsilon\left(S A S^{-1}\right)\right]^{2}-|\operatorname{tr} A|^{2} / n\right\}^{1 / 2}+|\operatorname{tr} A| / n .
$$

Theorem 5. Let $A$ be an $n \times n$ complex nonsingular matrix. Then

$$
[\rho(A)]^{2} \leqq\left[\epsilon\left(S A S^{-1}\right)\right]^{2}-(n-1)\left\{|\operatorname{det} A|^{2} /\left[\epsilon\left(S A S^{-1}\right)\right]^{2}\right\}^{1 /(n-1)}
$$

for any nonsingular $S$.

Proof. Let $\lambda_{M}$ be an eigenvalue of maximum modulus. As in Theorem 4,

$$
\left|\lambda_{M}\right|^{2} \leqq\left[\epsilon\left(S A S^{-1}\right)\right]^{2}-\sum_{i \neq M}\left|\lambda_{i}\right|^{2}
$$

An application of the arithmetic-geometric mean inequality yields 


$$
\left|\lambda_{M}\right|^{2} \leqq\left[\epsilon\left(S A S^{-1}\right)\right]^{2}-(n-1) \prod_{i \neq M}\left|\lambda_{i}\right|^{2 /(n-1)}
$$

But

$$
\begin{aligned}
\prod_{i \neq M}\left|\lambda_{i}\right|^{2 /(n-1)} & =\left(|\operatorname{det} A|^{2} /\left|\lambda_{M}\right|^{2}\right)^{1 /(n-1)} \\
& \geqq\left\{|\operatorname{det} A|^{2} /\left[\epsilon\left(S A S^{-1}\right)\right]^{2}\right\}^{1 /(n-1)},
\end{aligned}
$$

from which the result follows.

We observe that the quantity $\left[\epsilon\left(S A S^{-1}\right)\right]^{2}$ occurring in Theorems 4 and 5 may be replaced by the bound for it given by Theorem 3 . We use this fact in the discussion of the following numerical example which illustrates the various bounds. Let

$$
A=\left[\begin{array}{rrr}
2 & 3 & 2 \\
10 & 3 & 4 \\
3 & 6 & 1
\end{array}\right]
$$

Then $\rho(A)=11$ and $\left(\sum_{i=1}^{3}\left|\lambda_{i}\right|^{2}\right)^{1 / 2}=11.58$. The Ledermann bound [2] is 16.77.

The bound of Theorem 3 is 11.9 and, using this bound, we obtain from Theorem 4 the bound 11.3 and from Theorem 5 the bound 11.6.

California Institute of Technology

Pasadena, California

1. A. BRAUER, "The theorems of Ledermann and Ostrowski on positive matrices," Duke Math. J., v. 24, 1957, p. 265-274. MR 19, 7.

2. W. LeDERMANN, "Bounds for the greatest latent roots of a positive matrix," J. London Math. Soc., v. 25, 1950, p. 265-268. MR 12, 312.

3. A. Ostrowski, "Über Normen von Matrizen," Math. Z., v. 63, 1955, p. 2-18. MR 17, 228.

4. A. OSTROWSKI, "Bounds for the greatest latent roots of a positive matrix," J. London Math. Soc., v. 27, 1952, p. 254-256. MR 14, 126.

5. J. Todn, Ed., A Survey of Numerical Analysis, McGraw-Hill, New York, 1962. MR 24 * B1271.

6. R. S. Varga, Matrix Iterative Analysis, Prentice-Hall, Englewood Cliffs, N. J., 1962. MR $28 * 1725$. 